\title{
LA SOLIDARIDAD INTERGENERACIONAL. ENSAYO DE PERSPECTIVAS
}

Louis Roussel $^{1}$

La compasión se convierte en una variante del desprecio cuando da cuenta por sí sola de que nuestra relación con el prójimo excluye otros sentimientos, como el respeto, la admiración y la alegría.

Pascal BRUCKNER

En demografía, los pronósticos tienen mala reputación. Esto se debe a su perniciosa tendencia a ser desmentidos, muy a menudo por los hechos. Además, su presentación cuantitativa, a veces, oculta mal y, sin saberlo el propio autor, un prejuicio ideológico. El propósito es el de calcular los efectivos de la población, a largo plazo, pero se traduce solamente su ansiedad personal frente a la situación demográfica.

Los riesgos son muy parecidos en sociología (Berger, 1994). Los errores sobre lo que era considerado en el pasado como probable han sido tan numerosos y considerables en importancia, que el balance debería inducir a la renuncia de tales ejercicios. ¿Cuál será en el futuro la solidaridad entre generaciones? Las variables que intervienen en la elucidación del problema son demasiado numerosas para que se pueda encontrar una respuesta fiable. ¿No sería conveniente detenerse en lo que son actualmente estas relaciones? Ambas pre-

${ }^{1}$ INED, París.

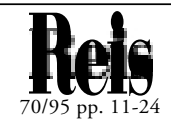


guntas son indisociables. Al analizar la situación demográfica actual, hay que buscar y tener en cuenta inercias que en el futuro pueden plantear problemas. En relación con la existencia de estos desafíos tenemos una relativa seguridad; en cambio, cómo la sociedad y las familias abordarán estos problemas, es una cuestión dudosa. Lo más que podemos esperar es un mejor conocimiento de los factores que intervendrán en tales decisiones.

\section{LOS FUNDAMENTOS DEMOGRAFICOS DE LOS NUEVOS PROBLEMAS}

La expresión "relaciones intergeneracionales» abarca un campo muy amplio. Aquí, nos limitaremos, directamente al menos, a las relaciones padres/hijos, y a las formas en que pueden presentarse en los diferentes momentos del ciclo familiar.

Lo que precisamente ha cambiado, a lo largo de estos treinta años, es el desglose del ciclo de vida debido al alargamiento de la coexistencia de las dos generaciones y a una segmentación más sutil de estas etapas. En la actualidad, se están desarrollando dos etapas nuevas, por generalización y extensión: la juventud y la primera parte de la jubilación. En realidad, los jóvenes han existido siempre, en el sentido que aquí entendemos, e igualmente las jubilaciones adelantadas, pero se trataba, frecuentemente, de situaciones minoritarias y breves. Actualmente, ambas etapas han cobrado una gran importancia, de modo que ahora se puede hablar de "edades» sociales concretas.

En primer lugar, consideramos la juventud como la edad que va de la mayoría legal a la autonomía efectiva en la relación con los padres. Cualquiera que sea el indicador consultado, no escapa a la evidencia el hecho de que, en dos décadas, esta situación ha pasado, en Francia, de los dieciocho a los veinticuatro años. Así, la proporción de los hombres jóvenes que conviven con sus padres hasta los veinticuatro años ha pasado del 32 por 100, en 1968, al 47 por 100, en 1990. Por su parte, las mujeres casadas a los veintidós años, en 1960 representaban el 75 por 100 de su generación, en tanto que actualmente no suponen más que el 29 por 100. Asimismo, la proporción de estudiantes en relación al conjunto de la generación de veintidós años era del 8 por 100 en 1968, y en 1990 se situaba alrededor del 30 por 100, de acuerdo con la información censal. He aquí, pues, una situación de dependencia, para la mayoría de la población, que se alarga probablemente una media de más de cinco años, después de la emancipación legal.

Es evidente que este aumento de jóvenes es debido, esencialmente, a dos razones contradictorias, pero ambas efectivas. La primera, es la prosperidad económica de los "gloriosos treinta" y la consiguiente posibilidad natural de proporcionar una mejor cualificación merced a unos estudios de mayor duración. Con posterioridad surge, sin lugar a dudas, otra razón, el paro, que se ha convertido en un factor determinante. Hasta finales de los años cincuenta, la mayor parte de los 
padres consideraban que su responsabilidad "alimentaria» terminaba, para los varones, con el servicio militar, y para las mujeres, con un matrimonio precoz; y en cualquier caso, para unos y otras, alrededor de los veintiún años. La prolongación de la juventud quedaba reservada a los estudiantes. En el futuro, aun en la hipótesis de un descenso sensible del paro, la necesidad de una fuerte preparación educativa, junto con el retraso de la edad al matrimonio, mantendrán, al menos para la mayoría, una etapa de juventud que, en algunos casos, se prolongará, a casi una decena de años, a partir de los dieciocho.

Además, se ha producido un segundo cambio en la antepenúltima etapa de la vida, la inserción, entre la jubilación y la auténtica vejez, de un largo período profesionalmente inactivo donde se prolonga la salud de la edad adulta o al menos la autonomía física. En 1970, la jubilación se producía, generalmente, hacia los sesenta y cinco años, con lo que quedaba entonces una esperanza de vida de trece años, por término medio. En la actualidad, la jubilación se inicia alrededor de los sesenta años, y a esta edad un hombre puede vivir una media de diecinueve años, habida cuenta de que el retroceso de la mortalidad se ha acelerado después de 1970. (Actualmente, la esperanza de vida a los sesenta años aumenta de dos meses por año).

Sin duda, en el curso de los próximos decenios podremos contemplar una ralentización de esta evolución; en el peor de los casos, se producirá una estabilización. Pero la inversión de la tendencia, salvo una catástrofe, es apenas concebible. Del mismo modo, a corto plazo, es poco probable un retraso sensible en la edad de la jubilación. La duración media de la vida después de la jubilación será, por consiguiente, alrededor de los diecinueve años para los hombres y de, al menos, veinticinco años para las mujeres, en los próximos veinte años.

Estos cambios han permitido a Ségolène Royal hablar de la «primavera de los abuelos». La expresión, reconfortante, puede ser incluso demasiado optimista, dado que esta relativamente larga etapa no puede ser considerada como un período homogéneo. El nuevo jubilado es todavía un adulto, se encuentra en una condición física mejor que la que tenían sus padres a esa edad. Pero llega un momento en el que la auténtica vejez se presenta en grados diferentes, y más o menos tardíamente aparece como alguna forma de invalidez. ¿A qué edad sobreviene? No se puede predecir, puesto que varía considerablemente, según los individuos. Para aquellos que no mueren precozmente o de repente existe una larga etapa de vida en la que, profesionalmente inactivos, cada uno permanece activo libremente, invirtiendo en ellos mismos, realizando proyectos durante largo tiempo aplazados, manteniendo o multiplicando las relaciones sociales, y estando más presentes que antes en las familias de sus hijos.

Ahora, la auténtica vejez es mucho más breve, por lo general. En este caso los ancianos se convierten en los hijos de sus hijos y están, al menos en términos afectivos, a su cargo. Lo están para las prestaciones, los cuidados, las salidas. En este sentido, es esencial responder a una pregunta: ¿El aumento de la esperanza de vida, a partir de los sesenta años, está acompañado de una duración igual del período de vida "sin incapacidad»? Si éste no fuese el caso, el 


\section{TABLA 1}

Variación de la esperanza de vida a los sesenta y cinco años, entre 1981 y 1991 (en años)

Francia

\begin{tabular}{|c|c|c|c|}
\hline & 1981 & 1991 & Evolución \\
\hline \multicolumn{4}{|l|}{ Hombres } \\
\hline 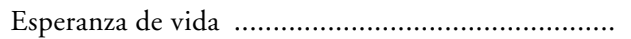 & 14,1 & 15,7 & $+1,6$ \\
\hline Esperanza de vida sin incapacidad grave .................. & 13,1 & 14,8 & $+1,7$ \\
\hline 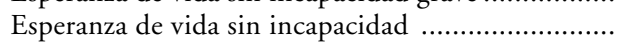 & 8,8 & 10,1 & $+1,3$ \\
\hline \multicolumn{4}{|l|}{ Mujeres } \\
\hline 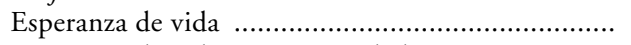 & 18,3 & 20,1 & $+1,8$ \\
\hline Esperanza de vida sin incapacidad grave .................... & 16,5 & 18,1 & $+1,6$ \\
\hline 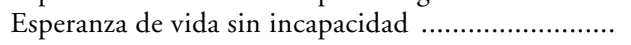 & 9,8 & 12,1 & $+2,3$ \\
\hline
\end{tabular}

FUENTES: Situations démographiques. Recensement de la population et Enquétes sur la santé. INSEE.

Cálculo: INSERM. Laboratoire d'épidemiologie et d'économie de la santé de Mompellier.

aumento probable de la esperanza de vida conduciría a una más larga duración del período de la autonomía disminuida. Afortunadamente, la hipótesis más verosímil no es ésta, al menos por el momento.

Pero, volviendo al tema de la prolongación de la esperanza de vida se advierte, que ha tenido un efecto muy importante sobre la pirámide de edades, desde el momento que ha modificado el peso respectivo de los grandes grupos de edad.

\section{TABLA 2}

División de la población por grandes grupos de edad (1960-1990-2020) Francia

\begin{tabular}{|c|c|c|c|}
\hline & 1960 & 1990 & 2020 \\
\hline $0-19$ & 32,3 & 27,6 & 22,6 \\
\hline $20-59$ & 51,0 & 53,3 & 51,3 \\
\hline 60-74 & 12,4 & 12,4 & 17,9 \\
\hline \multirow[t]{2}{*}{ 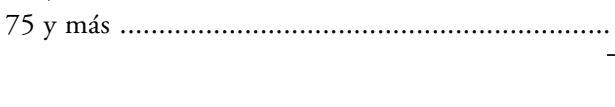 } & 4,3 & 6,7 & 8,2 \\
\hline & 100 & 100 & 100 \\
\hline
\end{tabular}

Fuente: 1960 y 1990, censo 2020, DINH, «Projections de la population de la France Metropolitaine», en Les Proyections Démmographiques, PUF, 1987. 
La estabilidad de los 60-74 años, entre 1960 y 1990, se explica por el paso de las generaciones, menos numerosas, nacidas en el curso de la primera guerra mundial. Por el contrario, el crecimiento de los mayores de setenta y cinco años, en el curso de esos tres decenios, ha sido muy importante. No obstante, el conjunto de los mayores de sesenta años, entre 1960 y el 2020, debería aumentar un 56 por 100; esto supone un cambio sensible que dará lugar a un aumento de las obligaciones financieras. La constatación más evidente en 1990 es el aumento en un 50 por 100 de los mayores de setenta y cinco años.

Hay otro factor interviniente, la mayor frecuencia del divorcio, que ha perturbado, en una parte significativa de la población, la solidaridad entre generaciones. Para simplificar, se puede decir que la media del índice actual de divorcios es de uno por cada tres matrimonios, aunque, en ciertos medios sociales y en el caso concreto de París, se produce un divorcio por cada dos matrimonios. Generalmente, el divorcio no interrumpe la solidaridad entre padres e hijos, pero la desequilibra, ya que ésta se refuerza con la madre, mientras se atenúa sensiblemente con el padre. Las encuestas recientes del INED muestran que, en un 40 por 100 de los casos, las relaciones entre padre e hijos se convierten, en el mejor de los casos, en irregulares y esporádicas.

Además, existe una última razón que pone en cuestión las solidaridades, es la crisis económica que ha dejado de mostrarse como coyuntural y se ha instalado como situación, con cifras elevadas de desempleo, especialmente entre los jóvenes.

Al mismo tiempo que ocurría todo esto, las demandas sociales aumentaban, en tanto que los recursos del Estado disminuían. De hecho, éste ha intentado desentenderse, de repente, de un buen número de obligaciones sociales otorgadas en tiempos de prosperidad; ha dejado de ser "estado de providencia» para ser percibido, cada vez más, como una «madre malvada». Habrá que encontrar una buena, ésta será la familia; que será exhortada, ya lo es, para ocupar los lugares abandonados por los poderes públicos. Esta familia, de la que se podía pensar en los años sesenta que se definiría cada vez más, solamente por sus funciones afectivas (Parsons, 1955), ahora se ha convertido en manifiestamente instrumental, investida de nuevas obligaciones, en particular, para con los hijos sin trabajo y los padres ancianos.

Las nuevas estructuras demográficas, el nuevo ciclo de vida, la movilidad matrimonial, la crisis económica prolongada, suponen datos nuevos para las relaciones intergeneracionales. Será necesario ver ahora, de manera más precisa, cuáles son los problemas que plantean.

\section{LOS PROBLEMAS}

Considerando, en primer lugar, las relaciones entre hijos y padres, se podría decir que son hasta cierto punto tan naturales que ninguna modifica- 
ción del contexto puede afectarlas. La solidaridad supone una fuerte dependencia afectiva recíproca y una dependencia material unilateral. Las prestaciones de los padres son visibles y medibles (Glaude, 1991), las de los hijos escapan de toda aritmética. Gracias a los hijos, la pareja se encuentra a menudo promovida a una dignidad efímera, pero muy gratificante, representar para otro ser la totalidad de lo real.

Como se ha mencionado, esta solidaridad está cada vez más afectada por el divorcio. El niño es confiado a su madre; esto no es la regla, pero sí la norma. La ruptura de la solidaridad conyugal va a constituir para los hijos un desafío, al necesitar una reorganización difícil de su economía afectiva. Que el divorcio no supone una prueba insalvable es algo generalmente admitido; que el divorcio es, en ciertos casos, una situación preferible a la inmersión en conflictos permanentes, es un punto de vista compartido por la mayoría de los psicólogos; pero que el divorcio sea para los hijos una ruptura fácil, una anécdota banal, es una cuestión a revisar. Se produce una disminución real de las relaciones con el padre, también con los abuelos paternos, y con toda esa rama de la familia. Más adelante se establecerán otras solidaridades con el nuevo cónyuge de la madre que podrían causar problemas, sobre todo, si los hijos no son muy pequeños. En esta situación las relaciones intergeneracionales de los hijos se modifican, se multiplican, se transforman, de hecho, no van muy lejos.

Cuando esta situación se convierte en estadísticamente importante, produce un efecto "perverso", en el sentido que Boudon manifiesta, es decir, un efecto colectivo no deseado. Nuestro modelo familiar, que es bilineal, con el mismo grado de solidaridad para con los dos linajes, se convierte cada vez más en matrilineal, desde el momento que todo matrimonio comporta desde su inicio (Théry, 1993) un riesgo importante de ruptura. Aunque parece que esta tendencia es acusada actualmente, la frecuencia del divorcio no es, por otra parte, más que una de las razones.

También es necesario hablar de las relaciones entre padres y adolescentes, pero no lo haremos porque los factores del cambio han sido más culturales que demográficos. Se sabe que la solidaridad es difícil para las dos partes, los adolescentes reclaman una mayor emancipación que a los padres no les parece conveniente, y los padres tienen el sentimiento de haber perdido toda su autoridad. Momento difícil, donde el medio familiar es esencial para el equilibrio de los adolescentes.

Detengámonos ahora en esta «nueva edad», denominada juventud. Es un período extraño hecho de independencia, ya que el hijo es mayor, y de dependencia, puesto que no puede asumir su autonomía económica. Libertad de derecho y dependencia de contratiempos y restricciones conviven en un tiempo de pruebas y experiencias, de obligada precariedad. Se vive, afectivamente y, sobre todo, profesionalmente, bajo el signo de lo provisional. 


\section{CUADRO 1}

Tasas de situaciones precarias entre los activos en función de la edad

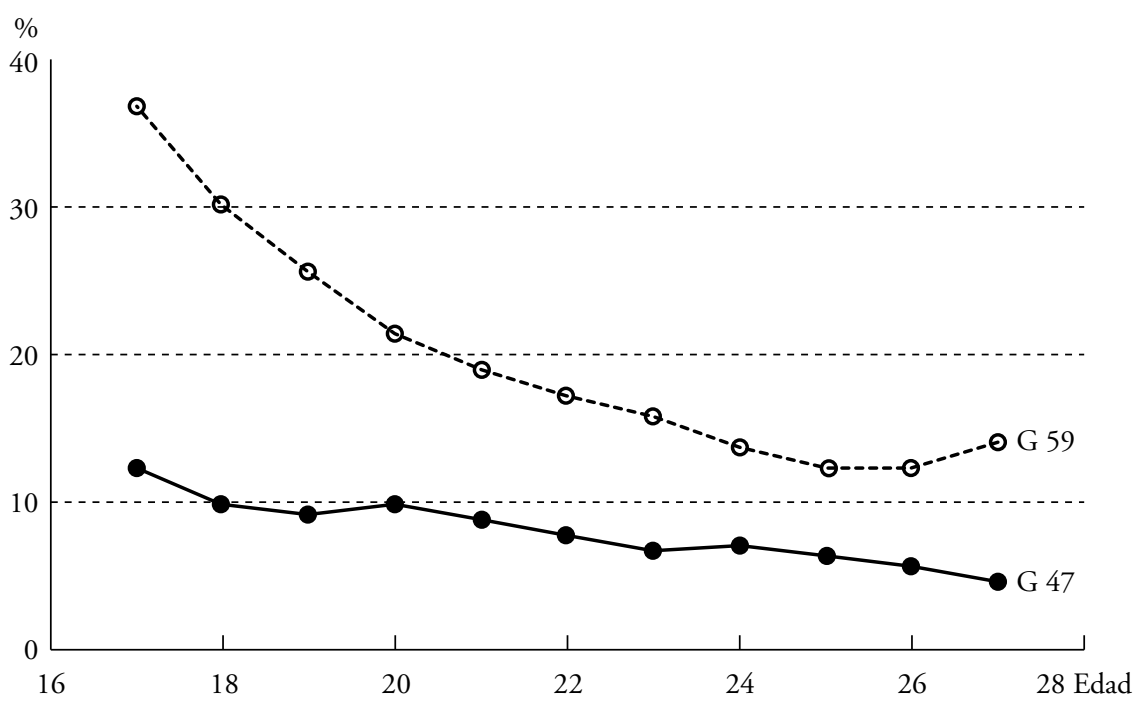

Fuente: F. Godard, La famille, affaire de générations, París, PUF, 1992, p. 162.

En este caso se trata de una población muy heterogénea, desde el punto de vista profesional, asalariados con contrato de duración limitada, desempleados, estudiantes; y en relación al estado matrimonial, solteros, cohabitantes, raras veces casados, de los cuales algunos disponen de una residencia personal y otros viven con sus padres.

Solamente una minoría ha dejado el hogar paterno; éstos reciben una ayuda financiera, regular o no, que les permite vivir. En este modelo, padres e hijos se visitan con frecuencia si la distancia entre sus residencias lo permite. Están encantados de verse; comen juntos de vez en cuando, todo está en regla, aceptado por ambas partes. Las ocasiones tensas son escasas, pero se producen, a pesar de todo. Los jóvenes pueden pensar que los padres subestiman sus deseos y los padres juzgar excesivas las exigencias de los jóvenes. Pero la distancia cotidiana atenúa las divergencias y hace más raras las discusiones. No es el mejor de los mundos, pero no se vive en un estado de malos entendidos permanentes. La fórmula supone, por tanto, una cierta calma para los padres, calma que amenaza el desempleo; aunque este acuerdo podría llegar a ser menos frecuente de lo que es ahora.

La otra fórmula más extendida, y la única practicable para muchos, es la permanencia de los jóvenes en el hogar paterno. Esta se prolonga, más allá de 
la mayoría de edad, en una familiaridad cotidiana donde, en principio, cada generación deberá encontrar su fórmula de vida, una con la presencia de los hijos en casa, la otra con la casi certeza de una cierta seguridad material. Pero la cuestión no es tan simple. Los hijos están en una situación ambigua, siendo adultos de derecho, siguen siendo menores económicamente. En cuanto a los padres, no queda muy claro el «estatus» de los servicios que otorgan, se supone ¿que es una obligación que la duración de esta etapa alarga, o una generosidad que releva a la obligación terminada? ¿El hijo tiene derecho siempre a «su casa» o no se encuentra en ella más que en calidad de huésped privilegiado? De hecho, los padres guardan el recuerdo de lo que se les dijo cuando ellos eran adolescentes: «El día en que te marches de casa, harás lo que quieras; mientras permanezcas aquí harás lo que nosotros queramos».

Así, pues, el tiempo de esta cohabitación es y será el de una solidaridad inevitable pero difícil. Ni los padres ni los hijos tienen el sentimiento de estar verdaderamente en «su casa». Los primeros podrán considerar que algunas veces se les trata como criados; los segundos pensarán que los padres pretextan su ayuda para reprimir una libertad en principio reconocida. De modo que los padres se sienten responsables, sin autoridad; los hijos, libres, sin medios. Las tensiones pueden multiplicarse; los conflictos permanecerán la mayoría de las veces latentes y se resolverán después de la marcha de los jóvenes. Se trata, por tanto, de una situación nueva, pero con todas las posibilidades de prolongarse en los próximos decenios.

Los conflictos provocarán excepcionalmente una ruptura, que se alargará durante largos períodos de desavenencia que la llegada de los nietos no logrará disipar. Cada una de las partes cree que su dignidad está en juego, punto en el cual se suele ser intransigente. Por tanto, no se puede pensar que la convivencia, prolongada más allá de la mayoría legal, será cotidianamente idílica y sin riesgos de ruptura.

Una vez que los hijos estén instalados, sus padres continuarán ayudándoles, en caso de necesidad o de inversiones importantes. En esta época de desempleo, será particularmente apreciada, como forma de asistencia, la utilización de la red de relaciones para encontrar una cierta posición o para recuperar una para los hijos. Pero, esta vez, los roles están mejor definidos; hay donadores y beneficiarios y, de todas formas, los nietos se convierten en excelentes mediadores.

Saltemos algunos años; los padres se han convertido en abuelos, tienen entre cincuenta y sesenta años. Con respecto a la situación actual, el número de mujeres activas a esta edad no deja de aumentar; por tanto, serán menos las que pueden ocuparse regularmente de sus nietos menores. Además, en estos momentos la precariedad del empleo del esposo retendrá en el trabajo a aquellas mujeres que en otro tiempo podrían haberse ocupado de los nietos debido a una jubilación anticipada. De modo que habrá que esperar algunos años para que la abuela alcance la edad de la jubilación. 
Cuando los abuelos llegan a estar disponibles, la mayoría aún conserva buena salud, y la llegada de la jubilación les permitirá alcanzar aún una mejor "forma». Todos disponen de tiempo libre que pueden invertir a su voluntad. Aman a sus hijos y nietos, se establece o, más bien, se desarrolla un intercambio, en apariencia al menos, asimétrico de bienes y servicios, signos de afecto, por un lado, y gratitud, por otro. De nuevo insistimos en que los nietos son excelentes mediadores. Se ven con frecuencia, se comparten las fiestas y se convive durante una parte de las vacaciones. Si se produce alguna tensión, proviene de una competencia real o imaginaria entre los padres del hijo y de la hija. No obstante, la autonomía residencial de cada hogar relativiza los malos entendidos. Ambas partes, padres e hijos adultos, se esfuerzan por evitar hablar de lo que «molesta»; los grandes temas se alejan a menudo de las conversaciones, política, religión y sobre todo la educación de los niños. Llega un momento en el cual los niños se convierten en adolescentes, la mayoría de los abuelos no son todavía ancianos, pero los servicios que prestan a la familia son menos frecuentes y ven relativamente poco a sus nietos. Pero, no obstante, están ahí, a menudo generosos, y siempre accesibles, en caso de necesidad urgente. El alargamiento y la generalización de este período que precede a la verdadera vejez, reaviva las relaciones entre las dos generaciones, no planteando verdaderos problemas.

Todo cambia cuando los padres pierden una parte de su autonomía. La dependencia se invierte de forma brusca o progresiva, no resulta una situación fácil para nadie. Los padres ancianos no reaccionan siempre de la misma manera. Algunos son exigentes y consideran correcto estar rodeados de cuidados y afectos por parte de sus hijos. ¿Es que acaso los padres no les han dedicado a ellos toda su vida? Pero éstos, realmente, no disponen de suficiente tiempo libre y no pueden consagrarse totalmente a sus padres, sorprendiéndose, en ocasiones, de lo poco realistas que son sus exigencias.

Otros padres, en cambio, son discretos y no esgrimen tener derecho al afecto y a los servicios de sus hijos, aunque no por ello dejarán de experimentar en ocasiones el sentirse abandonados. En cuanto a los hijos, las mujeres sobre todo, experimentan una cierta culpabilidad por no haber hecho por sus padres todo lo que anhelarían hacer por ellos.

Nos encontramos ante una situación nueva, y si como es plausible, por el momento, el alargamiento de la vida se acompaña de un igual alargamiento del período de incapacidad, ¿qué ha cambiado entonces? En realidad, existen varios factores nuevos que modifican y agravan sensiblemente las dificultades.

En primer lugar, el período de pérdida de autonomía se ha retrasado, llegando un momento en que la familia, frecuentemente, está compuesta por cuatro generaciones. Esta situación exige, evidentemente, una redistribución de los recursos disponibles, del tiempo, en especial. La nueva abuela, sobre todo si es todavía activa, tendrá problemas para satisfacer a todo el mundo. Se sentirá bombardeada por demandas diversas y a menudo incompatibles. El prestar mucho tiempo a su madre, enferma o incapacitada, sólo puede ser en perjuicio las otras generaciones que, en ocasiones, no dudarán en hacérselo 
saber. En este punto, la bisabuela corre el peligro de salir perjudicada. Estamos, pues, ante un elemento nuevo que se generalizará progresivamente.

El segundo factor es el de los efectos del divorcio. Recordemos, en primer lugar, el riesgo que supone para los abuelos paternos encontrarse de repente apartados de sus nietos. Esta ruptura se produce, generalmente, antes de la vejez, pero sus efectos se hacen sentir durante largo tiempo. Además, hay que insistir en otra consecuencia que aunque menos evidente es igualmente importante. En la medida en que la frecuencia del divorcio multiplica el número de mujeres solas a los cincuenta años, tendrán que soportar la presión cortés, pero firme y tenaz, para que se hagan cargo de la «anciana mamá». Esta es la solución más fácil para los demás, y sin duda satisfactoria para la persona mayor, pero que hace de esta mujer una verdadera cautiva.

Además, hay otro factor que modifica la situación con respecto a lo que era en los decenios precedentes, la crisis económica, cuyos efectos, el desempleo en particular, amenazan con prolongarse en el curso del próximo decenio. Sus consecuencias afectarán probablemente a los jubilados más que a los otros grupos de edades. En efecto, en los años sesenta la reivindicación principal de los asalariados era obtener una jubilación más prolongada en las mejores condiciones. Reivindicación que fue, por diversas razones, ampliamente satisfecha. El objetivo prioritario hoy en día es, evidentemente, la reducción del desempleo. En este sentido, los jubilados aparecen como los privilegiados, como el único grupo de edad que está generalmente al abrigo de la precariedad en base a recursos igualitarios, ligeramente superiores a los de las personas activas. No es necesario ser muy listo para imaginar que, de una manera u otra, los jubilados tienen algo que ver en una parte del deterioro general de la economía.

No es este el lugar para evaluar las formas que asumirá la redistribución de las obligaciones y las prestaciones sociales entre los grandes grupos de edades. Pero no es seguro que en el momento de la auténtica vejez los jubilados puedan beneficiarse de las ayudas que reciben hoy en día y, menos aún, que sus disminuidos recursos les permitan, lo que es ya hoy difícil, entrar en una institución especializada. No les quedará, pues, más recurso que sus hijos. El problema tampoco es nuevo, pero que corre el riesgo de agravarse.

Todos estos cambios podrían traducirse, a nivel social, en una «guerra entre generaciones». Las personas de edad avanzada considerarán los logros adquiridos, durante el período de la prosperidad, como un derecho inalienable, en tanto que los asalariados, desempleados o asalariados con riesgo de desempleo, considerarán a las personas mayores como los injustos beneficiarios de una situación pasada. Esto no ocurrirá sin que cambien las representaciones colectivas recíprocas. Los ancianos serán considerados como uno de los principales causantes del déficit publico, de la Seguridad Social, por ejemplo. Si la situación de crisis se mantiene, serán considerados como un obstáculo a la recuperación económica y su imagen social se devaluará.

¿Repercutirá esta guerra entre generaciones en las relaciones familiares? Puede ser, pero no es seguro. Somos lo bastante inconsecuentes como para, al 
mismo tiempo, deplorar lo que perjudique a nuestros padres y felicitarnos de lo que un justo correctivo deparará a su generación.

En resumen, la redistribución de las etapas de la vida y los cambios socioeconómicos que la acompañan han alargado el tiempo de convivencia entre las generaciones, aproximadamente un cuarto de siglo, y, sobre todo, el período de coexistencia en alrededor de medio siglo, lo cual ha multiplicado las ocasiones y las demandas de intercambios. Son los padres quienes hasta el umbral de su auténtica vejez ven aumentar sus obligaciones, en general. En contraposición, es probable que los hijos estén ya sobrecargados y se sobrecarguen aún más, con las responsabilidades que surjan en los últimos años de la vida de sus padres. En fin, esta familia de la que algunos pudieron pensar, hacia 1950, que iba a reducir sus funciones progresivamente a los intercambios afectivos, expresivos (Stoetzel, 1946; Parsons, 1955), se convierta ahora para todos en una institución instrumental que presta sus servicios, pero también los exige, en tanto dure la coexistencia de las dos generaciones. Puede que sea más gratificante que en otras ocasiones, pero su carga es también más difícil de soportar. Ante estas nuevas condiciones objetivas, ¿puede, por tanto, predecirse cuáles serán las reacciones futuras?

\section{POSIBLES REACCIONES A LAS NUEVAS CONDICIONES}

En las familias tradicionales las obligaciones de los hijos eran más pesadas que hoy en día; no sólo comportaban una ayuda a los padres mayores, sino también un gran aporte de fuerza de trabajo, a lo largo de lo que actualmente consideramos la etapa de juventud. Los padres, por su parte, alimentaban a los hijos y les transmitían un patrimonio. Pero esta solidaridad era considerada, sobre todo, como una obligación estatutaria; las cosas eran así no porque los hijos fuesen amables o porque los padres fueran generosos, sino porque unos eran los padres y los otros eran los hijos. Cada uno sabía lo que debía hacer, lo cual no significaba que cada cual cumpliera con su obligación. Todo el sistema estaba organizado en torno a la supervivencia como finalidad, aunque las personas mayores apenas contribuían a este objetivo. La obligación teórica apenas se mantenía por la utilidad social, y como bocas inútiles eran generalmente los «excluidos» de estas sociedades.

Actualmente, en nuestras familias las obligaciones estatutarias han dado paso, en gran medida, a los requerimientos afectivos. La solidaridad tiene un fundamento más sentimental; se cuida a los hijos no por deber, sino por amor; la fidelidad conyugal es menos un deber impuesto que una exigencia de la autenticidad del amor; las relaciones de pareja han dejado de establecerse en contra de los cónyuges; el divorcio, lógicamente, pervive.

A esta psicologización general de la solidaridad parece escapársele, al menos, un tipo de relación: la que une a padres e hijos. Una encuesta reciente 
(Riffault, 1994) mostraba que nuestros contemporáneos toman, hoy en día, sus decisiones, no en función de los principios establecidos a priori, sino en consideración de las consecuencias probables. Parecen ajustarse menos a una tabla del bien y del mal que a un balance prospectivo de las ventajas e inconvenientes de la elección. Dentro de esta actitud general, se produce una excepción, la solidaridad entre padres e hijos, que es contemplada independientemente de las circunstancias y considerada como incondicional.

Es una opinión común a todos los países de la Unión Europea. Pero, si ésta se correspondiese a la realidad de las prácticas, los intercambios objetivos en las obligaciones mutuas de las generaciones no podrían alterar las solidaridades que, de todas formas, permanecerían establecidas estatutariamente, y, por tanto, independientes de los sentimientos. Por tanto, estatutariamente, cada generación debería, en los diferentes momentos del ciclo familiar, asumir obligaciones, sin duda, más pesadas que antes, pero impuestas con la misma fuerza. ¿Qué importancia tendría entonces la pirámide de edades y la redefinición de las etapas de la vida?

Por otra parte, si esta respuesta, casi unánime, corresponde a una convicción general, ¿se traduce igualmente en un comportamiento unánime? Aquí sería preciso recordar la proporción importante de padres divorciados que renuncian a sus hijos, igualmente habría que evocar la situación de los abuelos ancianos que no reciben de sus hijos más que escasas visitas y manifestaciones un poco irrisorias de afecto. La distinción entre los momentos de fuerte solidaridad y los de relativa relajación permite aclarar un poco la cuestión.

Si se excluyen los casos de divorcio, las relaciones padres/hijos son intensas o fuertes desde el nacimiento de los hijos a la entrada de los padres en la vejez. ¿No podría explicarse esta solidaridad constante por el hecho de que durante este largo período se producen intercambios asimétricos aunque equilibrados entre las dos generaciones? Equilibrados, merced a una equidad, real o percibida como tal, en las prestaciones útiles de la mayoría de los padres, frente a los signos de afecto de la mayoría de los hijos, pequeños o adultos. En efecto, todo no puede reducirse, por parte de los padres, a las ayudas accesorias, y por parte de los hijos, a los signos de cariño. Se trata de dos maneras de expresar la ternura; dos maneras complementarias, donde cada parte encuentra su compensación. Tomando la palabra interés en un sentido amplio, se podría decir que cada generación tiene, durante todo este período, interés en mantener la solidaridad.

Queda por analizar la situación totalmente diferente de las personas de edad realmente avanzada. Se puede decir de manera esquemática que, en lo sucesivo, su cuenta está definitivamente «en rojo». Dicho de otro modo, el intercambio ya no está equilibrado, estas personas demandan más de lo que pueden dar; sobre todo, si no tienen ningún patrimonio, están totalmente desprovistos de recursos y sus exigencias quedan sin contrapartida. En la medida en la cual pueda garantizarse una ayuda domiciliaria, asalariada o prestada por los servicios sociales, la carga que corresponde a los hijos será bastante ligera y 
generalmente asumida. ¿Qué sucederá si estos ancianos se empobrecen aún más y el Estado reduce sus compromisos? Esta es una pregunta, evidentemente, muy difícil de contestar.

Se trata, pues, de una edad crucial donde el intercambio no juega ya ningún papel y en donde el argumento será que dicho intercambio está diferido y que los padres tienen un crédio bien ganado. Sin duda, pero ¿cómo se puede asegurar que serán respetados en una sociedad donde el largo plazo está desvalorizado? ¿Se podrá argüir que la compasión y la ternura son suficientes? Es éste, por supuesto, el caso más común, pero existe algún remedio eficaz contra la ingratitud o la indiferencia?

La vejez, época en donde la desigualdad de los intercambios es evidente, plantea claramente la cuestión sobre si la solidaridad entre las generaciones puede estar basada en el mutuo interés. ¿No hay, al menos, algún momento en el ciclo vital, donde se manifiesta la incapacidad del sentimiento y la utilidad del mantenimiento de la solidaridad? Si la familia contemporánea funcionara únicamente en base al sentimiento, el sistema no desembocaría en la exclusión de ciertos grupos, el primero de los cuales es el de las personas mayores.

En realidad, el problema es más general. Otros miembros de la familia pueden ser también despojados duramente del intercambio: los minusválidos, de alguna manera; los enfermos del SIDA y también, en estos tiempos de crisis, los económicamente excluidos del sistema. Si el sentimiento no constituye una garantía suficiente sobre cualquier otra seguridad incondicional, ¿se puede decir que se ha tocado fondo?

La encuesta anteriormente citada (Riffault, 1994), como otras, sobrevalora la importancia del respeto mutuo en las relaciones familiares y sobre todo en las conyugales. Probablemente, habría que reflexionar sobre el fundamento y las implicaciones de esta actitud. Puede ser que la palabra «respeto» signifique una requisito para el intercambio, un límite impuesto a los intereses de dicho intercambio que, en suma, permanecerá hasta que la racionalidad deje de unir a las personas.

En suma, la solidaridad intergeneracional supondrá una relación incondicional, aunque esté enmascarada la mayoría de las veces, o sea manifiesta, al menos con respecto a los padres de edad avanzada, dicha relación sólo podrá ser interrumpida por el abandono.

\section{CONCLUSION}

$\mathrm{Si}$, finalmente, es cierto que la familia impone la idea de una cierta incondicionalidad en las relaciones, habrá también que tener en cuenta la solidaridad social, la que mantiene unidos a los ciudadanos. En períodos de prosperidad, se admitirá, sin ninguna duda, que el interés individual reside en el reparto negociado de las obligaciones y los beneficios. La sociedad será como una gran empresa donde cada uno encuentra beneficios, incluso en el caso de que 
haya que hacer ciertos reajustes de vez en cuando. En este sistema, los perdedores seguirán existiendo: ayer, los pobres; hoy, los excluidos; su destino quedará en manos de las organizaciones de caridad. Dicho de otro modo, del sentimiento. Pero es posible imaginar que la solución de los problemas sociales sea finalmente dejada a lo que Pascal Bruckner (1994) llama «arbitraje del corazón». Si es cierto que en la familia el afecto supone y apela al respeto, y, simétricamente, en la sociedad la fraternidad reclama la justicia, ni en uno ni en otro caso podemos hacer de la economía un deber incondicional; a esto se le llama derecho.

\section{BIBLIOGRAFIA}

Berger, P. (1994): «¿Does sociology still make sense?», Revue Suisse de Sociologie, 1.

BRUCKNER, P. (1994): «L'arbitraire du coeur ou l'idéologie caritative», Esprit, marzo-abril 1994.

Glaude, M., y Montandier, M. (1991): «Une évaluation du coût direct de l'enfant de 1979 à 1989", Economie et Statisque, núm. 248.

GODARD, F. (1992): La famille, affaire de générations, París, PUF.

PARsons, T. (1955): Family, Socialization and Interation Process, Glencoe, Free Press.

Riffault, H. (1994): Les valeurs des Français, París, PUF.

Royal, S. (1987): Le printemps des grands-parents, París, Laffont.

Stoetzel, J. (1946): Sociologie et Démographie, Population, 1.

Théry, I. (1993): Le démariage, París, O. Jacob.

\section{RESUMEN}

Las transformaciones demográficas que afectan a una buena parte de la población, plantean problemas de todo tipo, pero especialmente de carácter social y económico. El alargamiento de la vida ha obligado a un replanteamiento de las etapas del ciclo vital y al establecimiento de nuevas formas en las relaciones intergeneracionales. Dentro de éstas la solidaridad juega un papel esencial. Solidaridad que se manifiesta, fundamentalmente, entre padres e hijos. De los padres hacia los hijos, durante la etapa de primera y segunda juventud, y solidaridad de los hijos hacia los padres, en la época de la vejez. Solidaridad que viene a cubrir, en buena medida, las deficiencias de un precario «estado del bienestar».

\section{ABSTRACT}

The demographic changes that affect a large part of the population involve a broad range of problems, particularly of an economic and social nature. Increased life expectancy has dictated the need for a new approach to the different phases of the life-cycle and the establishment of new forms of intergenerational relationship. In this context solidarity plays a central role. Solidarity is principally manifest between parents and children: from parents to children in the first and second phases of youth, and from children to parents during old age. To a large extent, this solidarity makes up for the shortfalls of a precarious welfare state. 